\title{
Constructive General Bounded Integral Control
}

\author{
Baishun Liu \\ Academy of Naval Submarine, Qingdao, China \\ Email: baishunliu@163.com
}

Received 1 June 2014; revised 5 July 2014; accepted 15 July 2014

Copyright (C) 2014 by author and Scientific Research Publishing Inc.

This work is licensed under the Creative Commons Attribution International License (CC BY). http://creativecommons.org/licenses/by/4.0/

c) (i) Open Access

\section{Abstract}

This note proposes a systematic and more generic method to construct general bounded integral control. It is established by defining three new function sets and citing two function sets to construct three kinds of general bounded integral control actions and integrators, resorting to a universal strategy to transform ordinary control into general integral control and adopting Lyapunov method to analyze the stability of the closed-loop system. A universal theorem to ensure regionally as well as semi-globally asymptotic stability is provided in terms of some bounded information, and even does not need exact knowledge of Lyapunov function. Its one feature is that the indispensable element used to construct the general integrator can be taken as any integrable function, which satisfies Lipschitz condition and the self excited integral dynamic is asymptotically stable. Another feature is that the method to construct general bounded integral control action is extended to a wider function set. Based on this method, the control engineers not only can choose the most appropriate control law in hand but also have more freedom to construct the bounded integral control actions and integrators, and then a high performance integral controller is more easily found. As a result, the generalization of the bounded integral control is achieved.

\section{Keywords}

General Integral Control, Nonlinear Control, Bounded Integral Control, General Nonlinear Integrator, Output Regulation

\section{Introduction}

In 2009, the idea of general integral control, which uses all available state variables to design the integrator, firstly was proposed by [1], which presented some general integrators and controllers. However, their justification was not verified by mathematical analysis. In 2012, general integral control design based on linear system theory was presented by [2], where the linear combination of all the states of dynamics was used as the integrator. 
The results, however, were local. The regional as well as semi-global results were proposed in [3], where the sliding mode manifold was used as the integrator, and then general integral control design was achieved by using sliding mode technique and linear system theory. In 2013, a class of nonlinear integrator, which was shaped by diffeomorphism, was proposed by [4], where feedback linearization technique was used to analyze the closed-loop system stability. General concave integral control was proposed in [5], where a class of concave function gain integrator is presented and the partial derivative of Lyapunov function is introduced into the integrator design. In consideration of the twinning of the concave and convex concepts, general convex integral control was proposed by [6], where the method to design the convex function gain integrator is presented and its highlight point is that the integral control action seems to be infinity but its factually is finite in time domain. Although general concave and convex integral control are all bounded integral control, one major limitation of them is that the indispensable element of the integrator is limited to the partial derivative of Lyapunov function, another is the function sets, which are used to design the general concave and convex integrator and integral control action, only were limited to two kinds of function sets. These two limitations become a serious obstruction to design a high performance integral controller. In addition, the generalization of the integrator and integral control action, which is achieved by defining two function sets, respectively, was proposed by [7], and its one drawback is that the integral control action could tend to infinity.

In consideration of the limitation of general concave and convex integral control, the aim of this paper is to propose a systematic and more generic method to construct general bounded integral control such that for a particular application, the control engineers not only can choose the most appropriate control law in hand but also have more freedom to construct the bounded integral control action and integrator. The main contributions are as follows: 1) three new function sets are defined, respectively; 2) three kinds of method to construct general bounded integral control action and integrator are proposed; 3) the indispensable element used to construct the integrator is not confined to the partial derivative of Lyapunov function [5] [6] and function set [7], which is used to construct the integrator, and can be taken as any integrable function, which satisfies Lipschitz condition and the self excited integral dynamic is asymptotically stable; 4) the function sets used to construct the bounded integral control action have a wider range of choice than the corresponding function sets proposed by [5]-[7]; 5) a class of positive define bounded gain function is introduced into the integrator, which provides the designer with additional degrees of freedom to improve the control performance; 6) exact knowledge of Lyapunov function is not necessary and it only needs to satisfy some bounded information; 7) by using Lyapunov method and LaSalle's invariance principle, a universal theorem to ensure regionally as well as semi-globally asymptotic stability is established. As a result, the generalization of the bounded integral control is achieved.

Throughout this paper, we use the notation $\lambda_{m}(A)$ and $\lambda_{M}(A)$ to indicate the smallest and largest eigenvalues, respectively, of a symmetric positive define bounded matrix $A(x)$, for any $x \in R^{n}$. The norm of vector $x$ is defined as $\|x\|=\sqrt{x^{T} x}$, and that of matrix $A$ is defined as the corresponding induced norm $\|A\|=\sqrt{\lambda_{M}\left(A^{T} A\right)}$.

The remainder of the paper is organized as follows. Section 2 describes the system under consideration, assumption, definition and proof of Lemma. Section 3 addresses the method to construct general bounded integral control. Conclusions are presented in Section 4.

\section{Problem Formulation}

Consider the following nonlinear system,

$$
\left\{\begin{array}{l}
\dot{x}=f(x, w)+g(x, w) u \\
y=h(x, w)
\end{array}\right.
$$

where $x \in R^{n}$ is the state, $u \in R^{m}$ is the control input, $y \in R^{m}$ is the controlled output, $w \in R^{l}$ is a vector of unknown constant parameters and disturbances. The function $f, g$ and $h$ are continuous in $(x, w, u)$ on the whole control domain $D_{x} \times D_{u} \times D_{w} \subset R^{n} \times R^{m} \times R^{l}$. Let $r \in D_{r} \subset R^{m}$ be a vector of constant reference. Set $\vartheta \equiv(r, w) \in D_{\vartheta}$ and $D_{\vartheta} \equiv D_{r} \times D_{w}$. We want to design a feedback control law $u$ such that $y(t) \rightarrow r$ as $t \rightarrow \infty$.

Assumption 1: For each $\vartheta \in D_{\vartheta}$, there is a unique pair $\left(x_{0}, u_{0}\right)$ that depends continuously on $\vartheta$ and satisfies the equations, 


$$
\left\{\begin{array}{l}
0=f\left(x_{0}, w\right)+g\left(x_{0}, w\right) u_{0} \\
y=r=h\left(x_{0}, w\right)
\end{array}\right.
$$

so that $x_{0}$ is the desired equilibrium point and $u_{0}$ is the steady-state control that is needed to maintain equilibrium at $x_{0}$, where $y=r$.

No loss of generality, we state all definitions, theorems and assumptions for the case when the equilibrium point is at the origin of $R^{n}$, that is, $x_{0}=0$.

Assumption 2: No loss of generality, suppose that the function $g(x, w)$ satisfies the following inequalities,

$$
\begin{gathered}
g(x, w)>g_{m}>0 \quad \forall w \in D_{w}, \quad \forall x \in D_{x} \\
\|g(x, w)-g(0, w)\| \leq l_{g}^{x}\|x\| \quad \forall w \in D_{w}, \quad \forall x \in D_{x}
\end{gathered}
$$

where $l_{g}^{x}$ is a positive constant.

Assumption 3: Suppose that there exists a control law $u_{x}(x)$ such that $x=0$ is an exponentially stable equilibrium point of the system (5) and the inequality (6) hold,

$$
\begin{gathered}
\dot{x}=f(x, w)-f(0, w)+g(x, w) u_{x}(x) \\
\left\|f(x, w)-f(0, w)+g(x, w) u_{x}(x)\right\| \leq l_{f}^{x}\|x\|
\end{gathered}
$$

and there exists a Lyapunov function $V_{x}(x)$ such that the following inequlities,

$$
\begin{gathered}
c_{1}\|x\|^{2} \leq V_{x}(x) \leq c_{2}\|x\|^{2} \\
\left\|\frac{\partial V_{x}(x)}{\partial x}\right\| \leq c_{4}\|x\| \\
\frac{\partial V_{x}(x)}{\partial x}\left(f(x, w)-f(0, w)+g(x, w) u_{x}(x)\right) \leq-c_{3}\|x\|^{2}
\end{gathered}
$$

hold for all $x \in D_{x}, \vartheta \in D_{\vartheta}$, where $l_{f}^{x}, c_{1}, c_{2}, c_{3}$ and $c_{4}$ are all positive constants.

For the purpose of this paper, it is convenient to introduce the following definitions and Lemmas. For the convenient comparison with the general concave and convex integral control, it is necessary to explain that the following Definition 1 and 2 was proposed by reference [5] [6], respectively.

Definition 1: $F_{\varphi}\left(a_{\varphi}, b_{\varphi}, x\right)$ with $a_{\varphi}>0,1 \geq b_{\varphi}>0$ and $x \in R^{n}$ denotes the set of all continuous differential increasing bounded functions [5] [8],

$$
\varphi(x)=\left[\begin{array}{llll}
\varphi_{1}\left(x_{1}\right) & \varphi_{2}\left(x_{2}\right) & \cdots & \varphi_{n}\left(x_{n}\right)
\end{array}\right]^{\mathrm{T}}
$$

such that

$$
\begin{gathered}
\varphi(0)=0, \\
\left|x_{i}\right| \geq\left|\varphi_{i}\left(x_{i}\right)\right| \geq b_{\varphi}\left|x_{i}\right|, \quad \forall x_{i} \in R:\left|x_{i}\right|<a_{\varphi} \\
a_{\varphi} \geq\left|\varphi_{i}\left(x_{i}\right)\right| \geq a_{\varphi} b_{\varphi}, \quad \forall x_{i} \in R:\left|x_{i}\right| \geq a_{\varphi} \\
1 \geq \mathrm{d} \varphi_{i}\left(x_{i}\right) / \mathrm{d} x_{i}>0 \quad \forall x_{i} \in R(i=1,2, \cdots, n) .
\end{gathered}
$$

where $|\cdot|$ stands for the absolute value.

Figure 1 depicts the region allowed for one component of functions belonging to function set $F_{\varphi}$. For instance, for all $x \in R$, hyperbolic tangent function, arc tangent function, Amosin function [8] and so on, all belong to function set $F_{\varphi}$.

Definition 2: $F_{\phi}\left(a_{\phi}, c_{\phi}, x\right)$ with $a_{\phi}>0, c_{\phi}>0$ and $x \in R^{n}$, denotes the set of all continuous differential increasing functions [6],

$$
\phi(x)=\left[\begin{array}{llll}
\phi_{1}\left(x_{1}\right) & \phi_{2}\left(x_{2}\right) & \cdots & \phi_{n}\left(x_{n}\right)
\end{array}\right]^{\mathrm{T}}
$$




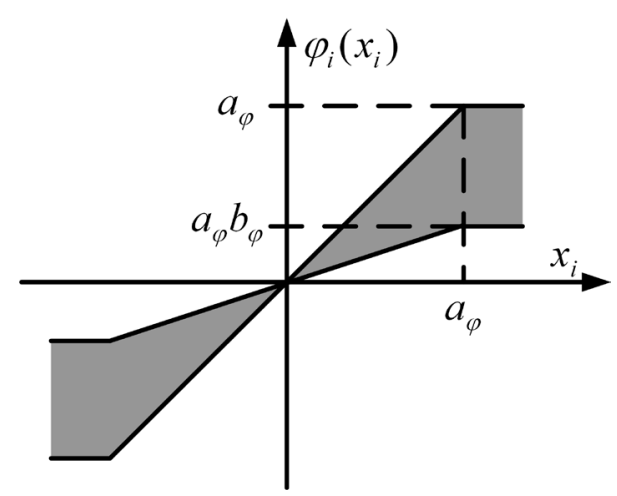

Figure 1. The region allowed for one component of functions belonging to function set $F_{\varphi}$.

such that

$$
\begin{gathered}
\phi(0)=0 \\
0<\left(\mathrm{d} \phi_{i}\left(x_{i}\right) / \mathrm{d} x_{i}\right)^{-1}<c_{\phi} \quad(i=1,2, \cdots, n)
\end{gathered}
$$

and given any $\varepsilon>0$, there exists a positive constant $a_{\phi}$ such that

$$
\left(\mathrm{d} \phi_{i}\left(x_{i}\right) / \mathrm{d} x_{i}\right)^{-1}<\varepsilon \quad \forall x_{i} \in R:\left|x_{i}\right|>a_{\phi} .
$$

where $|\cdot|$ stands for the absolute value.

Figure 2 describes an example curve and the region allowed for the derivative reciprocal of one component of functions belonging to function set $F_{\phi}$. For instance, for all $x \in R$, the functions, $\left(x+x^{5}\right) / 3.0, \sinh (x)$, $x+x^{3}$ and so on, all belong to function set $F_{\phi}$.

Definition 3: $F_{\psi}\left(a_{\psi}, b_{\psi}, x\right)$ with $a_{\psi}>0, b_{\psi}>0$, and $x \in R^{n}$ denotes the set of all continuous differential increasing functions,

$$
\psi(x)=\left[\begin{array}{llll}
\psi_{1}\left(x_{1}\right) & \psi_{2}\left(x_{2}\right) & \cdots & \psi_{n}\left(x_{n}\right)
\end{array}\right]^{\mathrm{T}}
$$

such that

$$
\begin{gathered}
\psi(0)=0, \\
\left|\psi_{i}\left(x_{i}\right)\right| \geq b_{\psi} \quad \forall x_{i} \in R:\left|x_{i}\right|>a_{\psi} \\
\mathrm{d} \psi_{i}\left(x_{i}\right) / \mathrm{d} x_{i}>0 \quad \forall x_{i} \in R \quad(i=1,2, \cdots, n) .
\end{gathered}
$$

Figure 3 depicts the example curves for one component of functions belonging to function set $F_{\psi}$. For instance, for all $x \in R$, the functions, $\operatorname{arcsinh}(x), \tanh (x), a x+b x^{3}$ with $a>0$ and $b>0, \sinh (x)$, and so on, all belong to function set $F_{\psi}$.

Definition 4: $F_{\beta}\left(a_{\beta}, c_{\beta}, x\right)$ with $a_{\beta}>0, c_{\beta}>0$ and $x \in R^{n}$ denotes the set of all continuous positive define bounded functions,

$$
\beta(x)=\left[\begin{array}{llll}
\beta_{1}\left(x_{1}\right) & \beta_{2}\left(x_{2}\right) & \cdots & \beta_{n}\left(x_{n}\right)
\end{array}\right]^{\mathrm{T}}
$$

such that

$$
c_{\beta} \geq \beta_{i}\left(x_{i}\right)>0 \quad \forall x_{i} \in R \quad(i=1,2, \cdots, n),
$$

and given any $\varepsilon>0$, there exists $a_{\beta}$ such that

$$
\beta_{i}\left(x_{i}\right)<\varepsilon \quad \forall x_{i} \in R:\left|x_{i}\right|>a_{\beta}(i=1,2, \cdots, n) .
$$

Figure 4 depicts the example curves and the region allowed for one component of functions belonging to 


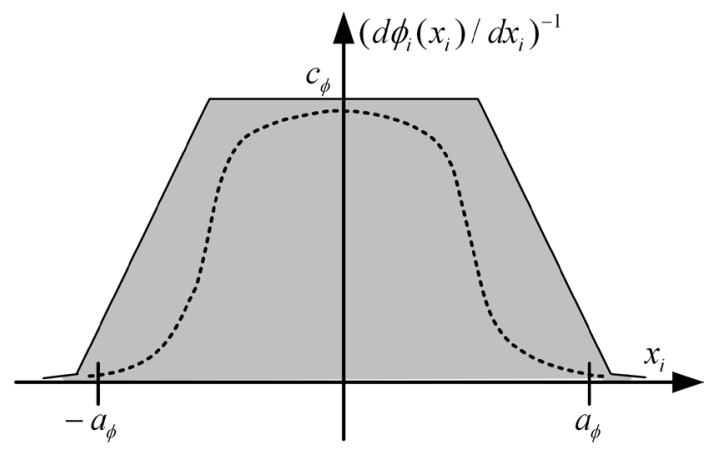

Figure 2. Example curve and the region allowed for the derivative reciprocal of one component of functions belonging to function set $F_{\phi}$.

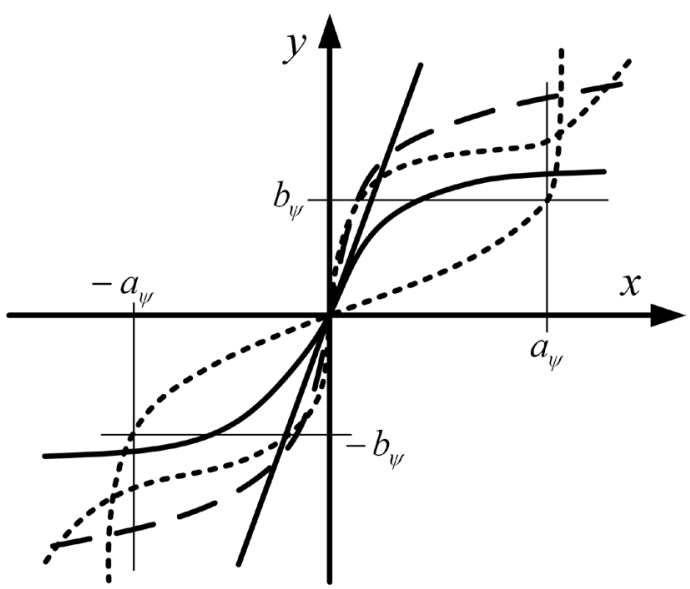

Figure 3. Example curves of functions belonging to function set $F_{\psi}$.

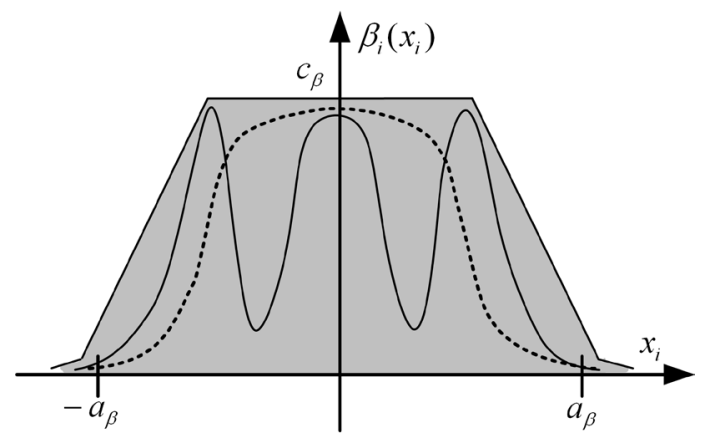

Figure 4. Example curves and the region allowed for one component of functions belonging to function set $F_{\beta}$.

function set $F_{\beta}$. For instance, for all $x \in R$, the functions, $\left(1+9 x^{2}\right) / \cosh ^{2}\left(x+3 x^{3}\right), 1 / \cosh ^{2}(x), 1 /\left(1+x^{2}\right)$, and so on, all belong to function set $F_{\beta}$.

Definition 5: $F_{v}(x)$ with $x \in D_{x} \subset R^{n}$ denotes the set of all integrable functions,

$$
v(x)=\left[\begin{array}{llll}
v_{1}(x) & v_{2}(x) & \cdots & v_{n}(x)
\end{array}\right]^{\mathrm{T}}
$$

such that 


$$
\|v(x)\| \leq l_{v}^{x}\|x\|,
$$

$x=0$ is an asymptotically stable equilibrium point of the self excited integral dynamic

$$
\dot{x}=-v(x) \text { for all } x \in D_{x} \text {. }
$$

where $l_{v}^{x}$ is a positive constant. For instance, for all $x \in R$, the functions, $x, x^{3}, x+x^{3}, \tanh (x), \sinh (x)$ and so on, all belong to function set $F_{v}$.

Lemma 1: Let $\mu(y) \in F_{\beta}$ or $\mu(y)=(\mathrm{d} \phi(y) / \mathrm{d} y)^{-1}$ with $y \in R$ or $\phi(y) \in F_{\phi}$, and then the function [6],

$$
y(t)=\int_{0}^{t} \mu(y(\tau)) \mathrm{d} \tau \quad \forall t \in[0, \infty)
$$

is a positive define bounded increasing function, that is, $0<y(t) \leq c_{\infty}$ for all $t \in[0, \infty)$, where $c_{\infty}$ is the limit of $y(t)$ as $t \rightarrow \infty$. Its proof consults the reference [6].

Lemma 2: Let $\mu(z) \in F_{\beta}$ or $\mu(z)=(\mathrm{d} \phi(z) / \mathrm{d} z)^{-1}$ with $z \in R, \phi(z) \in F_{\phi}$ and $v(x) \in F_{v}$, and then the function,

$$
z(t)=\int_{0}^{t} \mu(z(\tau)) v(x(\tau)) \mathrm{d} \tau \quad \forall t \in[0, \infty)
$$

is bounded, that is, $|z(t)| \leq \kappa_{\infty}$ for all $x \in D_{x}$ and $t \in[0, \infty)$, where $\kappa_{\infty}$ is a positive constant.

Proof: by definition of $z(t)$ and Definition 5, we have,

$$
|z(t)|=\left|\int_{0}^{t} \mu(z(\tau)) v(x(\tau)) \mathrm{d} \tau\right| \leq I_{v}^{x} \max _{x \in D_{x}}(\|x\|) \int_{0}^{t} \mu(z(\tau)) \mathrm{d} \tau
$$

Now, using Lemma 1, we obtain,

$$
|z(t)| \leq c_{\infty} I_{v}^{x} \max _{x \in D_{x}}(\|x\|)=\kappa_{\infty} .
$$

Thus, $z(t)$ is bounded, that is, $|z(t)| \leq \kappa_{\infty}$ for all $x \in D_{x}$ and $t \in[0, \infty)$.

Discussion 1: Comparing the two function sets $F_{\varphi}$ and $F_{\phi}$ proposed by [5] [6] with the function set $F_{\psi}$, it is no hard to see that although they all claim that the function is continuous differential increasing function, the main differences are as follows: the limiting conditions of the function set $F_{\psi}$ is less than the function sets $F_{\phi}$ and $F_{\varphi}$. Thus, the function set $F_{\psi}$ can completely includes the any functions belonging to the two function sets $F_{\varphi}$ and $F_{\phi}$.

Discussion 2: Comparing the function set [7], which was used to generalize the integral control action, with the function set $F_{\psi}$, the differences are the limiting condition about their derivatives, that is, the former demands $c_{\psi}>\mathrm{d} \psi_{i}\left(x_{i}\right) / \mathrm{d} x_{i}>0 \quad\left(c_{\psi}>0, \forall x_{i} \in R\right.$ and $\left.i=1,2, \cdots, n\right)$. However, the latter only requires

$\mathrm{d} \psi_{i}\left(x_{i}\right) / \mathrm{d} x_{i}>0$. Thus, the function set $F_{\psi}$ not only can completely include the any functions belonging to the function set proposed by [7] but also the functions belonging to the function set $F_{\psi}$ have a wider range of choice than the one proposed by [7].

Discussion 3: Comparing the function set [7], which was used to generalize the integrator, with the function set $F_{v}$, the differences are that: the former is defined by resorting to Mean Value Theorem, therefore, it requires that the function is differential. However, the latter is defined by designing a self excited integral dynamic, and only demands its origin is asymptotically stable, and then differentiability condition is removed. Thus, it is not hard to see that the function set $F_{v}$ not only can completely include the any functions belonging to the function set proposed by [7] but also the functions belonging to the function set $F_{v}$ have a wider range of choice than the one proposed by [7].

Discussion 4: It is obvious that the bound of function, $z(t)$, which is obtained by Lemma 2, is too conservative and even is not of interest. The situation, however, is not as bad as it might seem. As shown by Figure 2 and Figure 3, we can use $a_{\beta}$ or $a_{\phi}$ as its approximate value in practice, corresponding to $\varepsilon$ small enough.

\section{Constructive Method}

In general, integral controller comprises three components: the stabilizing controller, integral control action and integrator. Thus, a general integral controller can be given as, 


$$
\left\{\begin{array}{l}
u=u_{x}(x)-K_{\sigma} \Phi(\sigma) \\
\dot{\sigma}=(\mathrm{d} \Phi(\sigma) / \mathrm{d} \sigma)^{-1} \mu(\sigma) v(x)
\end{array}\right.
$$

where

$u_{x}(x)$ is an ordinary control law;

$K_{\sigma}$ is a positive define diagonal matrix;

$\Phi(\sigma)=\left[\begin{array}{lllll}\Phi_{1}\left(\sigma_{1}\right) & \Phi_{2}\left(\sigma_{2}\right) & \cdots & \Phi_{m}\left(\sigma_{m}\right)\end{array}\right]^{\mathrm{T}}$ is a continuous differential increasing function with $\Phi(0)=0$;

$\mu(\sigma)=\left[\begin{array}{llll}\mu_{1}\left(\sigma_{1}\right) & \mu_{2}\left(\sigma_{2}\right) & \cdots & \mu_{m}\left(\sigma_{m}\right)\end{array}\right]^{\mathrm{T}}$ is a positive constant vector or positive define vector function;

$v(x)$ belongs to function set $F_{v}$;

$$
\dot{\sigma}_{i}=\left(\mathrm{d} \Phi_{i}\left(\sigma_{i}\right) / \mathrm{d} \sigma_{i}\right)^{-1} \mu_{i}\left(\sigma_{i}\right) v_{i}(x) \quad i=1,2, \cdots, m .
$$

Thus, substituting (10) into (1), obtain,

$$
\left\{\begin{array}{l}
\dot{x}=f(x, w)+g(x, w) u_{x}(x)-g(x, w) K_{\sigma} \Phi(\sigma) \\
\dot{\Phi}(\sigma)=\mu(\sigma) v(x)
\end{array}\right.
$$

By Assumption 1 and choosing $K_{\sigma}$ to be nonsingular and large enough, and then set $\dot{x}=0$ and $x=0$ of (11), obtain,

$$
g(0, w) K_{\sigma} \Phi\left(\sigma_{0}\right)=f(0, w)
$$

Therefore, we ensure that there is a unique solution, $\sigma_{0}$, and then $\left(0, \sigma_{0}\right)$ is a unique equilibrium point of the closed-loop system (11) in the control domain of interest. At the equilibrium point, $y=r$, irrespective of the value of $w$.

Now, the design task is to provide methods to construct the bounded integral control action and integrator in the control law (10) such that $K_{\sigma} \Phi(\sigma)$ is bounded and $\left(0, \sigma_{0}\right)$ is an asymptotically stable equilibrium point of the closed-loop system (11) in the control domain of interest. To achieve this objective, the methods can be summarized as follows:

Method 1: If we choose $\Phi(\sigma) \in F_{\varphi}$, and then by definition of $F_{\varphi}$, it is easy to know that the integral control action is bounded for all $\sigma \in R^{m}$. Thus, $\mu(\sigma)$ can be taken as any positive define bounded vector function or positive constant vector, that is, $0<\mu_{i}\left(\sigma_{i}\right) \leq c_{\beta}$ with $\sigma_{i} \in R$ and $i=1,2, \cdots, m$. Consequently, we have,

$$
\begin{gathered}
\|\Phi(\sigma)\| \leq c_{\sigma} \sqrt{m} \quad\left(c_{\sigma}=a_{\varphi}\right), \text { and } \\
\left(\mathrm{d} \Phi_{i}\left(\sigma_{i}\right) / \mathrm{d} \sigma_{i}\right) \mu_{i}\left(\sigma_{i}\right) \leq c_{\beta}=c_{\Phi} \quad\left(c_{\Phi}>0\right) .
\end{gathered}
$$

As a result, the generalization of the general concave integral control is achieved.

Method 2: If we choose $\Phi(\sigma) \in F_{\phi}$, and then design $\mu(\sigma)$ such that

$$
\begin{gathered}
\left(\mathrm{d} \Phi_{i}\left(\sigma_{i}\right) / \mathrm{d} \sigma_{i}\right)^{-1} \mu_{i}\left(\sigma_{i}\right) \in F_{\beta}, \\
\left(\mathrm{d} \Phi_{i}\left(\sigma_{i}\right) / \mathrm{d} \sigma_{i}\right) \mu_{i}\left(\sigma_{i}\right) \leq c_{\Phi} \quad\left(c_{\Phi}>0\right), \text { and } \\
\mu_{i}\left(\sigma_{i}\right) \in F_{\beta}
\end{gathered}
$$

hold for all $\sigma_{i} \in R$ and $i=1,2, \cdots, m$. Thus, by Lemma 1 and 2, it is easy to know that this kind of integral control action is bounded in time domain, that is,

$$
\|\Phi(\sigma)\| \leq c_{\sigma} \sqrt{m}
$$

where

$$
c_{\sigma}=\gamma_{x} \max _{i \in m}\left(\Phi_{i}\left(c_{\infty}^{i}\right)\right),
$$




$$
\begin{gathered}
\gamma_{x}=\max _{x \in D_{x}}(\|v(x)\|) \leq l_{v}^{x} \max _{x \in D_{x}}(\|x\|), \\
c_{\infty}^{i}=\lim _{t \rightarrow \infty} \int_{0}^{t}\left(\mathrm{~d} \Phi_{i}\left(\sigma_{i}(\tau)\right) / \mathrm{d} \sigma_{i}(\tau)\right)^{-1} \mu_{i}\left(\sigma_{i}(\tau)\right) \mathrm{d} \tau, \text { and } \\
i=1,2, \cdots, m .
\end{gathered}
$$

As a result, the generalization of the general convex integral control is achieved.

Method 3: If we choose $\Phi(\sigma) \in F_{\psi}$, constructive general bounded integral control can be divided into two cases: 1) if $\Phi(\sigma)$ is bounded, and then $\mu(\sigma)$ can be taken as any positive define bounded vector function or positive constant vector. The condition for $\Phi(\sigma)$ is the same as Method 1;2) if $\Phi(\sigma)$ is unbounded for all $\sigma \in R^{m}$, and then $\mu(\sigma)$ needs to be designed like Method 2. The condition for $\Phi(\sigma)$ is the same as Method 2. It is obvious that this is a more generic method to construct general bounded integral control because the function set used to construct the bounded integral control action has a wider range of choice than the corresponding function sets proposed by [5]-[7]. Moreover, it is worth noting that $\mu(\sigma)$ can be designed like Method 2 when $\Phi(\sigma)$ is bounded.

In addition, it is convenient to introduce the variable, $a_{\Phi}$, which is equal to $a_{\varphi}, a_{\phi}$ and $a_{\psi}$, respectively, corresponding to the above three kinds of choices of the function $\Phi(\sigma)$.

Based on the control law $u_{x}(x)$ and three kinds of integral control actions and integrators above, the following theorem can be established.

Theorem 1: Under Assumptions 1 - 3, if there exists a positive define diagonal matrix $K_{\sigma}$ such that the following inequality,

$$
\lambda_{m}\left(g_{m} K_{\sigma} \Phi\left(a_{\Phi}\right)\right) \geq\|f(0, w)\|
$$

and the inequality (20) hold, and then $\left(0, \sigma_{0}\right)$ is an exponentially stable equilibrium point of the closed-loop system (11). Moreover, if all assumptions hold globally, and then it is globally exponentially stable.

Proof: To carry out the stability analysis, we consider the following Lyapunov function candidate,

$$
V\left(x, \Phi(\sigma)-\Phi\left(\sigma_{0}\right)\right)=V_{x}(x)+z^{T} P_{z} z
$$

where

$$
\boldsymbol{P}_{z}=\left[\begin{array}{ll}
P_{x} & P_{12} \\
P_{21} & P_{\sigma}
\end{array}\right], \quad z=\left[\begin{array}{c}
x \\
\Phi(\sigma)-\Phi\left(\sigma_{0}\right)
\end{array}\right],
$$

$P_{z}$ is a positive define $(n+m) \times(n+m)$ matrix;

$P_{x}$ is a $n \times n$ matrix; $P_{\sigma}$ is a $m \times m$ matrix;

$P_{12}$ is a $m \times n$ matrix, $P_{12}=P_{21}^{T}, P_{12} g_{m} K_{\sigma}>0$.

Obviously, Lyapunov function candidate (14) is positive define. Therefore, our task is to show that its time derivative along the trajectories of the closed-loop system (11) is negative define, which is given by,

$$
\begin{aligned}
\dot{V}\left(x, \Phi(\sigma)-\Phi\left(\sigma_{0}\right)\right)= & \dot{V}_{x}(x)+z^{T} P_{z} \dot{z}+\dot{z}^{T} P_{z} z \\
= & \frac{\partial V_{x}(x)}{\partial x} \dot{x}+x^{T} P_{x} \dot{x}+\dot{x}^{T} P_{x} x+x^{T} P_{12} \dot{\Phi}(\sigma)+\dot{\Phi}^{T}(\sigma) P_{21} x \\
& +\dot{\Phi}^{T}(\sigma) P_{\sigma}(\Phi(\sigma)-\Phi(\sigma))+(\Phi(\sigma)-\Phi(\sigma))^{T} P_{\sigma} \dot{\Phi}(\sigma) \\
& +\dot{x}^{T} P_{12}\left(\Phi(\sigma)-\Phi\left(\sigma_{0}\right)\right)+\left(\Phi(\sigma)-\Phi\left(\sigma_{0}\right)\right)^{T} P_{21} \dot{x}
\end{aligned}
$$

Substituting (12) into (11), we obtain,

$$
\begin{aligned}
\dot{x}= & f(x, w)+g(x, w) u_{x}(x)-g(x, w) K_{\sigma} \Phi(\sigma) \\
= & f(x, w)-f(0, w)+g(x, w) u_{x}(x) \\
& -(g(x, w)-g(0, w)) K_{\sigma} \Phi(\sigma) \\
& -g(0, w) K_{\sigma}\left(\Phi(\sigma)-\Phi\left(\sigma_{0}\right)\right)
\end{aligned}
$$


Now, by the above three kinds of choices of the function $\Phi(\sigma)$, we have,

$$
\|\dot{\Phi}(\sigma)\|=\|(\mathrm{d} \Phi(\sigma) / \mathrm{d} \sigma) \mu(\sigma) v(x)\| \leq c_{\Phi} l_{v}^{x}\|x\|
$$

Substituting (16) into (15), and using (3), (4), (6), (8), (9), (17) and $\|\Phi(\sigma)\| \leq c_{\sigma} \sqrt{m}$, we obtain,

$$
\begin{aligned}
\dot{V}\left(x, \Phi(\sigma)-\Phi\left(\sigma_{0}\right)\right)= & \dot{V}_{x}(x)+z^{T} P_{z} \dot{z}+\dot{z}^{T} P_{z} z \\
& \leq-\rho_{x}^{x}\|x\|^{2}+\rho_{x}^{\sigma}\|x\|\left\|\Phi(\sigma)-\Phi\left(\sigma_{0}\right)\right\| \\
& -\rho_{\sigma}^{\sigma}\left\|\Phi(\sigma)-\Phi\left(\sigma_{0}\right)\right\|^{2}
\end{aligned}
$$

where

$$
\begin{gathered}
\rho_{x}^{x}=c_{3}-2 l_{f}^{x}\left\|P_{x}\right\|-c_{\Phi} l_{v}^{x}\left(\left\|P_{12}\right\|+\left\|P_{21}\right\|\right) \\
-\left(c_{4}+2\left\|P_{x}\right\|\right) c_{\sigma} l_{g}^{x} \sqrt{m}\left\|K_{\sigma}\right\|, \\
\rho_{x}^{\sigma}=\left(c_{4}+2\left\|P_{x}\right\|\right)\left\|g(0, w) K_{\sigma}\right\|+2 c_{\Phi} I_{v}^{x}\left\|P_{\sigma}\right\| \text { and } \\
+\left(l_{f}^{x}+c_{\sigma} l_{g}^{x} \sqrt{m}\left\|K_{\sigma}\right\|\right)\left(\left\|P_{12}\right\|+\left\|P_{21}\right\|\right), \\
\rho_{\sigma}^{\sigma}=\lambda_{m}\left(\left(g_{m} K_{\sigma}\right)^{T} P_{12}\right)+\lambda_{m}\left(P_{21} g_{m} K_{\sigma}\right) .
\end{gathered}
$$

and then inequality (18) can be rewritten as,

$$
\dot{V}\left(x, \Phi(\sigma)-\Phi\left(\sigma_{0}\right)\right) \leq-\eta^{T} Q \eta
$$

where

$$
\boldsymbol{\eta}=\left[\begin{array}{c}
\|x\| \\
\left\|\Phi(\sigma)-\Phi\left(\sigma_{0}\right)\right\|
\end{array}\right], \quad \boldsymbol{Q}=\left[\begin{array}{cc}
\rho_{x}^{x} & -0.5 \rho_{x}^{\sigma} \\
-0.5 \rho_{x}^{\sigma} & \rho_{\sigma}^{\sigma}
\end{array}\right] .
$$

The right-hand side of the inequality (19) is a quadratic form, which is negative define when

$$
\rho_{x}^{x} \rho_{\sigma}^{\sigma}-0.25 \rho_{x}^{\sigma} \rho_{x}^{\sigma}>0
$$

Using the fact that Lyapunov function (14) is a positive define function and its time derivative is a negative define function if the inequalities (13) and (20) hold, we conclude that the closed-loop system (11) is stable. In fact, $\dot{V}=0$ means $x=0$ and $\sigma=\sigma_{0}$. By invoking LaSalle's invariance principle [9], it is easy to know that the closed-loop system (11) is asymptotically stable.

Discussion 5: Compared to general convex and concave integral control [5] [6], it is easy to see that this paper is not a simple extension of them but proposes a systematic and more generic method to construct general bounded integral control. The main progresses are as follows: 1$)$ the indispensable element $v(x)$ used to construct the integrator can be taken any functions belonging to function set $F_{v}$ and is not confined to the partial derivative of Lyapunov function $\partial V_{x}(x) / \partial x$, which is used to construct the integrator in [5] [6]; 2) a positive define bounded gain function $\mu(\sigma)$ is introduced into the integrator, which can be used to improve the integral control performance; 3 ) a class of new function set $F_{\beta}$ is defined, and then the method to construct general bounded integral control action and integrator is extended to a wider function set $F_{\psi}$. As a result, this is a fire new and more generic method to construct general bounded integral control action and integrator; 4) we need not exact knowledge of Lyapunov function $V_{x}(x)$ and only need it satisfy some bounded information. Moreover, if the partial derivative of Lyapunov function is attached into the function $v(x)$, the stability conditions can be relaxed. All these mean that the control engineers have more freedom to design the integrator and bounded integral control action, and then a high performance integral controller is more easily found.

Discussion 6: Compared to the generalization integrator and integral control action proposed by [7], the main differences are as follows: 1) the integrators proposed by [7] are unattached with the integral control action. However, the integrators presented here are all educed by differentiating the nonlinear function, which is used to produce the integral control action; 2) the integral control actions proposed by [7] could tend to infinity. However, the integral control actions proposed here are all bounded. This means that this kind of integral control can 
devote its mind to counteract the unknown constant uncertainties and filter out the other action, and then actuator saturation is easy to be removed in practice; 3) a positive define bounded gain function $\mu(\sigma)$ is introduced into the integrator, which provides the designer with additional degrees of freedom to improve the integral control performance; 4) as mentioned at Discussion 2 and 3, the function sets $F_{v}$ and $F_{\psi}$ used to construct the integrator and integral control action, respectively, all have a wider range of choice than the corresponding function sets proposed by [7].

Remark 1: From the statement above, It is obvious that: First, five function sets for constructing general bounded integral control action is enumerated; Second, three general methods to construct the bounded integral control action are proposed; Final, a universal theorem to ensure regionally as well as semi-globally asymptotic stability is established. Under the domination of this theorem, all of them synthesize a systematic and more generic method to construct general bounded integral control together. Consequently, for a particular application, the control engineers not only can choose the most appropriate control law in hand but also have more freedom to design the bounded integral control action and integrator, and then a high performance integral controller is more easily found.

\section{Conclusion}

This paper is not a simple extension of general convex and concave integral control but proposes a systematic and more generic method to construct general bounded integral control. The main contributions are as follows: 1) three new function sets are defined, respectively; 2) three kinds of method to construct general bounded integral control action and integrator are proposed; 3) the indispensable element used to construct the integrator is not confined to the partial derivative of Lyapunov function [5] [6] and function set [7], which is used to construct the integrator, and can be taken as any integrable function, which satisfies Lipschitz condition and the self excited integral dynamic is asymptotically stable; 4 ) the function sets used to construct the bounded integral control action has a wider range of choice than the corresponding function sets proposed by [5]-[7]; 5) a class of positive define bounded gain function is introduced into the integrator, which provides the designer with additional degrees of freedom to improve the control performance; 6) exact knowledge of Lyapunov function is not necessary and it only needs to satisfy some bounded information; 7) by using Lyapunov method and LaSalle's invariance principle, a universal theorem to ensure regionally as well as semi-globally asymptotic stability is established. As a result, the generalization of the bounded integral control is achieved.

\section{References}

[1] Liu, B.S. and Tian, B.L. (2009) General Integral Control. Proceedings of the International Conference on Advanced Computer Control, Singapore, 22-24 January 2009, 136-143. http://dx.doi.org/10.1109/ICACC.2009.30

[2] Liu, B.S. and Tian, B.L. (2012) General Integral Control Design Based on Linear System Theory. Proceedings of the 3rd International Conference on Mechanic Automation and Control Engineering, 5, 3174-3177.

[3] Liu, B.S. and Tian, B.L. (2012) General Integral Control Design Based on Sliding Mode Technique. Proceedings of the 3rd International Conference on Mechanic Automation and Control Engineering, 5, 3178-3181.

[4] Liu, B.S., Li, J.H. and Luo, X.Q. (2014) General Integral Control Design via Feedback Linearization. Intelligent Control and Automation, 5, 19-23. http://dx.doi.org/10.4236/ica.2014.51003

[5] Liu, B.S., Luo, X.Q. and Li, J.H. (2013) General Concave Integral Control. Intelligent Control and Automation, 4, 356361. http://dx.doi.org/10.4236/ica.2013.44042

[6] Liu, B.S., Luo, X.Q. and Li, J.H. (accepted) General Convex Integral Control. International Journal of Automation and Computing.

[7] Liu, B.S. (submitted) On the Generalization of Integrator and Integral Control Action. International Journal of Modern Nonlinear Theory and Application.

[8] Kelly, R. (1998) Global Positioning of Robotic Manipulators via PD Control plus a Class of Nonlinear Integral Actions. IEEE Transactions on Automatic Control, 43, 934-938. http://dx.doi.org/10.1109/9.701091

[9] Khalil, H.K. (2007) Nonlinear Systems. 3rd Edition, Electronics Industry Publishing, Beijing, 126-128, 482-484. 
Scientific Research Publishing (SCIRP) is one of the largest Open Access journal publishers. It is currently publishing more than 200 open access, online, peer-reviewed journals covering a wide range of academic disciplines. SCIRP serves the worldwide academic communities and contributes to the progress and application of science with its publication.

Other selected journals from SCIRP are listed as below. Submit your manuscript to us via either submit@scirp.org or Online Submission Portal.
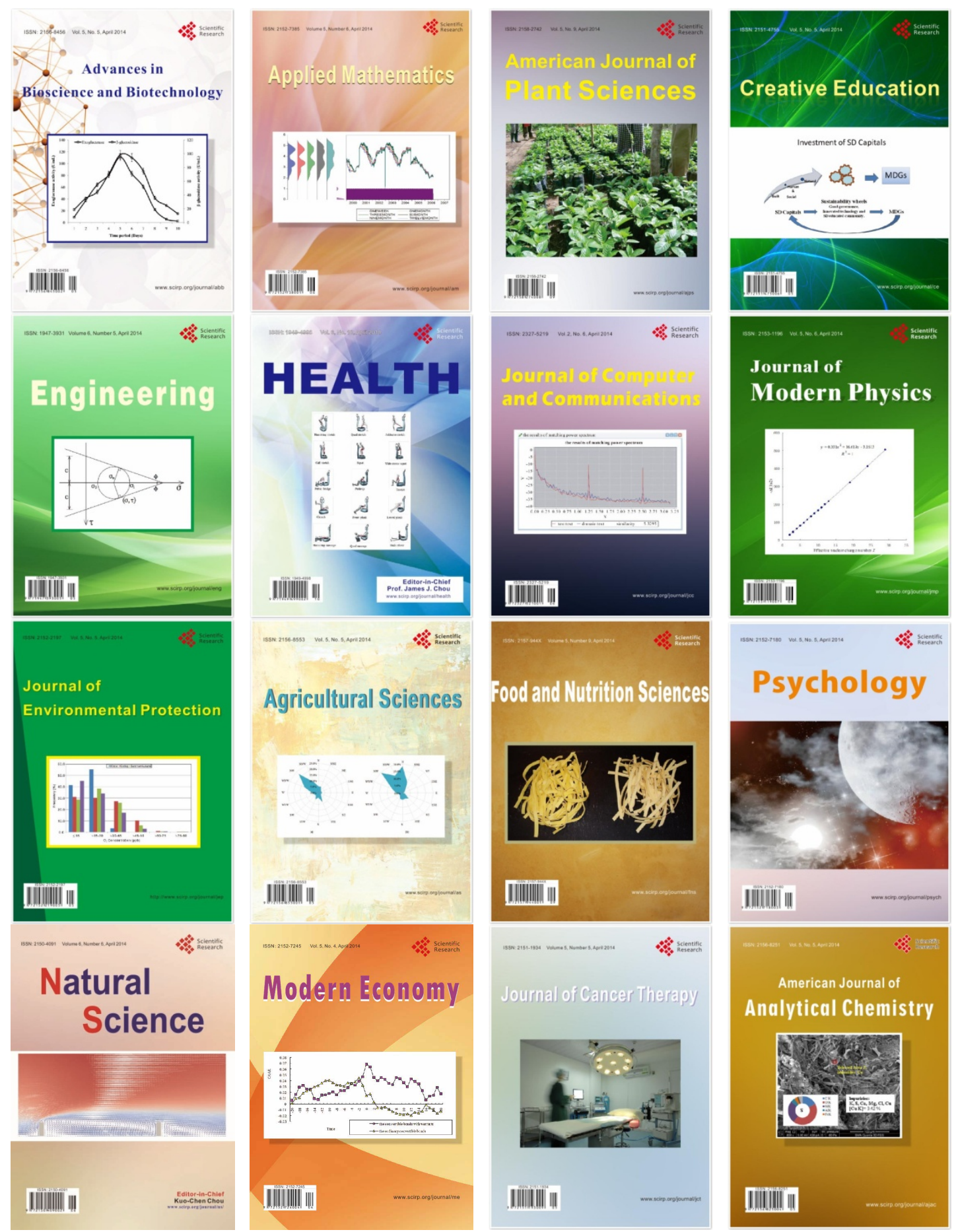